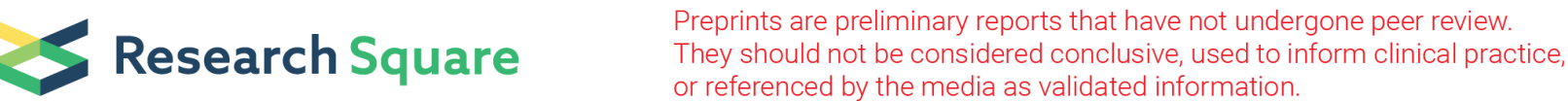

\section{Impact of integrative open dialogue about complementary alternative medicine. A phase II randomized controlled trial}

Mette Stie ( $\square$ mette.stie@rsyd.dk)

Sygehus Lillebalt Vejle Sygehus https://orcid.org/0000-0002-5254-9359

Charlotte Delmar

Aarhus University: Aarhus Universitet

Birgitte Nørgaard

University of Southern Denmark: Syddansk Universitet

Lars Henrik Jensen

Sygehus Lillebælt Vejle Sygehus: Sygehus Lillebalt Vejle Sygehus

\section{Research Article}

Keywords: Adverse events, cancer, complementary alternative medicine, consultation, communication, integrative, oncology, open dialogue, quality of life

Posted Date: May 12th, 2021

DOI: https://doi.org/10.21203/rs.3.rs-426226/v1

License: (9) This work is licensed under a Creative Commons Attribution 4.0 International License. Read Full License

Version of Record: A version of this preprint was published at Cancers on February 14th, 2022. See the published version at https://doi.org/10.3390/cancers14040952. 


\section{Abstract}

Complementary alternative medicine (CAM) may reduce the symptom burden of side effects to antineoplastic treatment but also cause new side effects and non-adherence to conventional treatment. The aim of this randomized controlled study was to investigate the impact of integrative open dialogue about CAM (IOD-CAM) on cancer patients' health and quality of life (QoL). Patients undergoing curative or palliative antineoplastic treatment were randomly assigned to standard care (SC) plus IOD-CAM or SC alone. A nurse specialist facilitated IOD-CAM in one or two sessions. The primary endpoint was the frequency of grade 3-4 adverse events (AE) eight weeks after enrollment. Secondary endpoints were frequency of grade 1-4 AE and patient reported QoL, psychological distress, perceived information, attitude towards and use of CAM 12 and 24 weeks after enrollment. Survival was analyzed post-hoc.

Fifty-seven patients were randomized to IOD-CAM and 55 to SC. No significant difference in frequency of grade 3-4 AEs was shown between the two groups eight weeks after enrollment. The same applied to grade 1-4 AE and QoL, psychological distress, and perceived information 12 and 24 weeks after enrollment. However, a tendency towards better QoL, improved survival, and lower level of anxiety was found in the IOD-CAM group.

IOD-CAM is not superior to $S C$ in reducing frequency of AEs in patients undergoing oncology treatment. IOD-CAM does not compromise patient safety; it may reduce psychological stress, and improve QoL and overall survival. Further research on the effect of IOD-CAM on emotional well-being and overall survival is warranted.

\section{Introduction}

Worldwide, increasing rates of cancer patients use complementary alternative medicine (CAM) as an adjunct to conventional treatment and care [1-3]. A systematic review reported CAM use among $75 \%$ of breast cancer patients undergoing chemotherapy [4]. Among patients undergoing treatment for colorectal cancer, a Danish study has shown that $49.9 \%$ use CAM [5]. Studies including patients with various types of cancer have found a prevalence of CAM use of $39.1 \%$ [6] and $60.3 \%$ [2].

There is no evidence that CAM has the potential to cure or affect the cancer disease, but some studies suggest that specific types of CAM are relevant as supportive therapies. Acupressure and acupuncture reduce nausea and pain [7], aromatherapy alleviates sleep and anxiety disorders [8], and massage, yoga, mindfulness, and meditation have shown to increase quality of life ( $Q \circ L)$ and reduce stress and fatigue [9]. CAM has also shown effective in relieving fear, fatigue, and depression [10] and enhancing hope [2], self-care, self-control, and empowerment $[11,12]$. However, the level of evidence ranges from high to low, and some CAMs include a potential risk of interaction with conventional medicine [13-15]. Therefore, to ensure patient safety and high quality care, it is crucial to integrate open dialogue about CAM between health professionals and patients in daily oncology care $[16,17]$. 
The open dialogue about CAM increases patient engagement, patient-centered communication, and higher clinician [18] and patient satisfaction [19]. It addresses patient stress and uncertainty, reduces exposure to misleading information, and enhances the patient-physician relationship, which is paramount in delivering high-quality care [20]. However, despite obvious safety, health, and QoL implications, open dialogue about CAM is not integrated in oncology care $[16,21]$. Consequently, the best method for integrating the dialogue has not yet been established. Researchers propose that an open dialogue about CAM within the field of oncology should rely on an integrative approach. This includes a healing oriented approach viewing and respecting patients as whole and unique physical, emotional, social, and spiritual beings with values, knowledge, preferences, and beliefs. Based on patient perspectives and evidence, CAM-information and counselling should be provided alongside conventional cancer care [22-26], but it remains unclear whether an integrative open dialogue about CAM (IOD-CAM) actually affects patients' safety, health, and QoL. The primary aim of this phase II randomized controlled trial was therefore to investigate whether IOD-CAM is superior to standard care (SC) in reducing frequency of adverse events in patients undergoing oncology treatment. Moreover, we hypothesized that patients participating in IODCAM would report improved QoL, reduced anxiety and depression, and higher level of information compared to patients receiving SC alone. The study was designed to provide knowledge on how to conduct a safe, high-quality IOD-CAM in oncology care.

\section{Method}

\section{Trial design}

This phase II, parallel group, randomized controlled trial compared the effectiveness of IOD-CAM with SC in reducing adverse events (AE) in patients undergoing oncology treatment and care. The study was prospectively registered with ClinicalTrials.gov (NCT03857776) and approved by the Danish Data Protection Agency through the Region of Southern Denmark (19-4309). According to the Committee on Health Research Ethics, their approval of the study was not required (15/42744). The protocol was not amended during the study, and since it did not involve any risks to the patients, no interim analysis was done. The study is reported according to the CONSORT guidelines [27].

\section{Setting}

The study was conducted at the Oncology Outpatient Clinic, Vejle Hospital, University Hospital of Southern Denmark, between April 2019 and July 2020. The Department of Oncology offers treatment and care to adult patients with breast, gynecological, prostate, pulmonary, colorectal, anal, and pancreatic cancer. There are around 57,000 outpatient visits to the department each year with 23,000 radiotherapy fractions and 9,300 chemotherapy and immunotherapy treatments administered.

\section{Participants}


The inclusion criteria were $\geq 18$ years of age, diagnosis of primary cancer or recurrence within the last three months, planned antineoplastic treatment (chemotherapy, immunotherapy and/or antibody therapy), realistic plan of at least two months of treatment, and life expectancy of six months. The ability to read and speak Danish was required. The exclusion criterion was participation in other trials interfering with the intervention or data collection. Eligible participants were informed and invited to participate in the study by health professionals prior to the first cycle of treatment in the outpatient clinic. Randomization onto the study was based on written and orally informed consent.

\section{Intervention group (IOD-CAM)}

In addition to SC, patients in the intervention group participated in one or two sessions of IOD-CAM facilitated by a nurse specialist. A primary caregiver participated, if preferred by the patient. The guideline for IOD-CAM presented in Figure 1 was inspired by the Andrew Weil Center for Integrative Medicine, University of Arizona and Schofield et al.'s recommendations [26, 28].

\section{Control group (SC)}

Patients randomized to the control group received SC and referral to www.kabcancer.dk, a website presenting research based information about potential effects and outcomes of CAM. SC was defined as oncology treatment and care, including antineoplastic drugs at the outpatient clinic. SC also involved continuous assessment of the patients' performance status, side effects, and symptoms, which were managed by specialist doctors and nurses.

\section{Randomization}

Based on written, orally informed consent, and subsequent baseline assessment, the patients were randomly assigned to either SC plus IOD-CAM or SC alone. Randomization 1:1 was computerized using Research Electronic Data Capture (REDCap) [29]. The study nurse informed the patients about the allocation. Patients randomized to IOD-CAM received a letter providing the date, time, and place for the IOD-CAM session and guidance for preparation. Patients randomized to SC received a pamphlet referring to the website www.kabcancer.dk.

Due to the nature of the intervention, neither patients nor staff were blinded to the allocation, but patients were strongly encouraged not to disclose the allocation status at the follow-up registration of adverse events.

\section{Outcome measures and data collection}


The primary outcome measure was the frequency of grade 3-4 AEs eight weeks after enrollment. The frequency of grade 0-4 AEs 12 and 24 weeks after enrollment were secondary outcome measures. At each follow up $(8,12$ and 24 weeks), patients' AEs were registered by a specialist nurse according to the Common Terminology Criteria for Adverse Events (CTCAEv5) [30]. We have registered the severity of 15 common AEs, i.e. dry mouth, oral mucositis, vomiting, nausea, constipation, diarrhea, pain, peripheral motor neuropathy, peripheral sensory neuropathy, fatigue, fever, febrile neutropenia, infections, hospitalization, and general discomfort. The secondary outcome measures included patient reported QoL, level of depression and anxiety, and perception of received information 12 and 24 weeks after enrollment.

To assess QoL, the validated European Organization for Research and Treatment of Cancer Quality of Life Questionnaire (EORTC QLQ C30) [31] was applied. It includes five functional scales, nine symptom scales and two global QoL scales. Patients' perception of information received was assessed using EORTC QLQ-INFO25 [32]. It consists of 26 items organized in four hypothesized scales; information about the disease, medical tests, treatment and other services, and eight single items. The level of depression and anxiety was assessed using the Hospital Anxiety and Depression Scale (HADS), which is a selfassessment scale composed of 14 items on two subscales assessing anxiety and depression symptoms in the past week [33]. The patients' use of and attitude towards CAM 24 weeks after enrollment was also measured. All questionnaires were administered electronically.

\section{Statistical methods}

The study is a randomized phase II screening trial [34] with a risk of type-1 error at 0.10 and a power of 0.80 . It was hypothesized that $25 \%$ of the patients in the IOD-CAM group would have grade 3-4 AEs eight weeks after enrollment compared to $50 \%$ in the SC group. Under these circumstances, 92 patients were required. To account for dropouts, the total number of patients to be enrolled was 106 .

The two groups were compared in all primary analyses. Demographic data are presented as counts (n) and proportions (\%), respectively, with means and standard deviations (SD). Chi-square test and Fisher's exact test was applied to detect differences between the two groups in relation to AEs. The EORTC QLQ C30 and INFO25 scores were reported as means with confidence intervals, and HADS scores as medians with $25^{\text {th }}-75^{\text {th }}$ percentiles. Comparison of the two groups relied on Student's t-test or Mann Whitney's U test.

P-values are reported to two decimal places. For the primary endpoint, two-sided p-values were used with a 0.10 level of significance. A professional academic statistician blinded to the study group assignment conducted all analyses.

\section{Results}

Of 454 patients screened for eligibility, 256 were invited to participate in the study. The remaining 198 were not invited due to restricted time resources. A total of 144 declined participation due to lack of 
interest $(n=53)$, lack of personal resources $(n=42)$, too many extra visits $(n=34)$, other reasons $(n=8)$, and administrative failure $(n=7)$. There were no significant differences in terms of age, sex, and cancer diagnosis between the decliners and those randomized (data not shown). In total, 112 patients were randomly assigned to IOD-CAM $(n=57)$ and SC $(n=55)$ (Figure 2). The groups were comparable in terms of baseline demographics, clinical characteristics, and use of and attitude towards CAM (Table 1).

The response rate in the study was high with $87 \%$ and $98 \%$ at $1^{\text {st }}$ follow-up ( 8 weeks), $94 \%$ and $86 \%$ at $2^{\text {nd }}$ follow-up ( 12 weeks), and $88 \%$ and $89 \%$ at $3^{\text {rd }}$ follow-up (24 weeks) for IOD-CAM and SC, respectively. (See Figure 2).

Patients in the IOD-CAM group participated in 0-4 consultations with the nurse specialist; four did not show up to the consultation due to lack of energy, 49 participated in one consultation, two had two consultations, and one patient had four consultations. In one patient, data on the number of consultations was lost.

At baseline, the two groups were comparable in terms of frequency of adverse events, QoL, depression and anxiety, and perceived information. During follow-up, the measurement scores over time was similar in the two groups.

\section{Adverse events}

Eight weeks after enrollment, no significant difference between the IOD-CAM and SC group was found for any type of grade 3-4 AE. The same applied to the follow-up after 12 and 24 weeks. Pooling of data at the patient level did not change this. Regarding grade 1-2 AEs the only statistically significant differences in the follow-up period were at eight weeks with nausea ( 28.9 vs $52.1 \%, p=0.02)$, mouth dryness $(60.0 \%$ vs $41.7 \%, p=0.03)$, and sensory neuropathy ( $44.4 \%$ vs $27.7 \%, p=0.05)$ in the IOD-CAM and SC groups, respectively (See Table 2).

\section{Quality of life}

There was no significant difference in QoL between the IOD-CAM and SC group at the 12 and 24-week follow-up (Figure 3). Similarly, and with only few exceptions, no significant differences were found withingroups during follow-up. From baseline to 12 weeks, a significant difference was shown in the SC group in one function and two symptoms. Social functioning declined by 9.65 points ( $95 \% \mathrm{Cl}$ : -18.59 to -0.71 ), diarrhea increased by 10.53 points (95\% Cl: 2.31 - 18.74) and nausea and vomiting increased by 6.41 points (95\% Cl: 0.96 - 11.86). Within the IOD-CAM group fatigue increased by 6.80 points (95\% Cl: 0.36 13.24). At 24 weeks, a significant difference was only shown within the IOD-CAM group. Physical functioning improved by 5.24 points ( $95 \% \mathrm{Cl}$ : 0.03 - 10.44), role functioning decreased by $9.13(95 \% \mathrm{Cl}$ : -17.47 to -0.78$)$ and diarrhea by 10.32 points ( $95 \% \mathrm{Cl}:-17.86$ to -2.77$)$. A remarkable increase of 4.63 
points (95\% Cl: -0.68 - 9.94) in emotional functioning was shown at 24 weeks in the IOD-CAM group. No statistically significant differences were found within the SC group at the third follow-up (Figure 3).

\section{Depression and anxiety}

As shown in Figure 4, there was no statistical difference in HADS median scores between the two groups during follow-up. A slightly difference in median anxiety score was observed at the second follow-up with 3 ( $25^{\text {th }}-75^{\text {th }}$ percentile: $\left.2-8\right)$ and $6.5\left(25^{\text {th }}-75^{\text {th }}\right.$ percentile: $\left.3-9\right)$ in the IOD-CAM and SC group, respectively. With respect to the depression score, the SC group reported a median score at $3\left(25^{\text {th }}-75^{\text {th }}\right.$ percentile: $\left.1-7\right)$ and the IOD-CAM group reported a median score at $2\left(25^{\text {th }}-50^{\text {th }}\right.$ percentile: $\left.1-4\right)$ at $2^{\text {nd }}$ follow-up.

\section{Level of perceived information}

In general, the INFO25 median scores were lower in the SC group compared to the IOD-CAM group during follow-up indicating that IOD-CAM might be superior to SC in providing sufficient information. However, the differences were not statistically significant. See supplementary Figure 5.

\section{Attitude towards and use of CAM}

No significant differences were found in the patients' attitude towards CAM, neither at baseline nor at the third follow-up (24 weeks). At baseline, 22 (40.7\%) and 20 (37.0\%) patients in the SC group reported a positive or very positive attitude towards CAM, respectively. At the third follow-up this had changed to 12 (31.6\%) and 16 (42.1\%), respectively. In the IOD-CAM group 33 (58.9\%) and 11 (19.6\%) patients reported a positive or a very positive attitude towards CAM, respectively, which changed to $23(53.5 \%)$ and $9(20.9 \%)$ at the third follow-up.

The use of CAM in the SC group changed from 40 (74.1\%) patients at baseline to $27(62.8 \%)$ at the third follow-up (24 weeks). Conversely, in the IOD-CAM group the use of CAM slightly increased from 35 (62.5\%) at baseline to $27(62.8 \%)$ at the third follow-up. The differences were not statistically significant.

\section{Explorative outcomes}

\section{Overall survival}

Survival tended to be higher in the IOD-CAM group compared to the SC group with an area under the curve (AUC) of $0.902(95 \% \mathrm{Cl}: 0.85-0.95)$ and $0.837(95 \% \mathrm{Cl}: 0.77-0.91)$, respectively. Thus, patients in 
the IOD-CAM lived in average $0.064(95 \% \mathrm{Cl}$ : -0.022 - 015) years longer than did the patients in the SC group, $\mathrm{P}=0.14$ (Supplementary Figure 6).

\section{Discussion}

In this study, 53 of 57 allocated to IOD-CAM completed the intervention and we consider it feasible to integrate in standard oncology care. The high completion rate could be an expression of patients' substantial need for reliable information and counselling about CAM as an integrated part of oncology care [35-37].

We found no significant difference in grade 3-4 AEs between the IOD-CAM and SC group after 8, 12 and 24 weeks of follow-up. Hence, IOD-CAM did not prove superior to SC in reducing the frequency of grade 34 AEs. We found a statistically significant difference at eight weeks in three grade 1-2 AEs. The frequency of nausea was lower in the IOD-CAM group and mouth dryness and sensory neuropathy was lower in the SC group. We found a statistically significant increase of nausea and vomiting in the SC group on the EORT QLQ C30- scale, although this was at the 12-week follow-up. In addition, diarrhea increased significantly in the SC group at 12 weeks and decreased significantly in the IOD-CAM group at 24 weeks on the EORTC QLQ C30 scale. However, no significant difference in diarrhea was found on the CTCAEv5 scale. Although not consistent, but supportive of similar studies (38), these findings indicate that IODCAM has potential in alleviating nausea, vomiting and diarrhea, which is clinically relevant, since these frequent $A E s$ associated with great concern in patients undergoing antineoplastic treatment [38, 39].

Overall, IOD-CAM does not compromise patient safety, which is an important finding. In-depth interviews have shown that health professionals are reluctant to discuss CAM due to skepticism as to its efficacy and safety [40]. Learning that IOD-CAM does not compromise safety may render health professionals more comfortable in discussing the issue with the patients.

We found a tendency towards better QoL with IOD-CAM, especially at the 24-week follow-up, which is in line with other studies [41]. A possible explanation of this delayed effect may be that the first phase of the antineoplastic treatment is the most burdensome. Another reason might lie in the fact that the IOD-CAM supports patients in becoming active participants in their own healing and health. Patients may feel more encouraged and focused on improving their QoL when the first phase of treatment is completed [41].

Congruent with other studies [36, 42], we found that IOD-CAM tends to be superior to SC in reducing patients' distress and in particular patients' anxiety. Studies have shown a high level of anxiety among patients who seek CAM counseling because they explore every possible treatment option [21]. IOD-CAM may thus reduce patients' level of anxiety because it includes counseling on both conventional treatment and CAM options. In addition, IOD-CAM is an open dialogue viewing and respecting patients as whole and unique physical, emotional, social, and spiritual beings with values, knowledge, preferences, and beliefs. Other researchers found that genuinely approaching patients as whole and unique persons leads to emotional well-being $[43,44]$. Finally, patients in the IOD-CAM group may have reported better 
emotional QoL and lower level of anxiety because their use of CAM tended to be higher than in the SC group. Use of CAM has in itself shown to improve patients' emotional well-being [45].

Interestingly, survival tended to be better in the IOD-CAM group. Combined with the lack of differences in adverse events, it could be speculated that the supportive care from IOD-CAM increases adherence to anti-cancer therapy and thus increases survival. Data collection on the proportion of anti-cancer treatment provided during the follow-up period was not part of the study.

\section{Strengths and limitations}

Strengths of this trial include the prospective, randomized design with a control group and the systematic data collection using validated patient-reported questionnaires and standardized classifications and registrations of $\mathrm{AE}$. However, we acknowledge that the study has some important limitations. Due to the phase II design, the study was not powered to fully assess the effect of IOD-CAM, and the nature of the intervention did not allow for blinding of patients and data collectors, which may have introduced bias. Moreover, the complexity of the intervention makes it difficult to determine which and how the elements of the intervention exert their potential effect. The majority of the patients had a positive attitude towards CAM and used CAM prior to enrollment. Although interest or use of CAM was not an inclusion criterion, the sampling frame might not be representative of patients who are not interested in discussing or using CAM. The study was conducted in a single cancer center, which might limit the generalizability of the results to other care settings and populations.

\section{Conclusion}

Integration of IOD-CAM into daily oncology care is feasible. IOD-CAM was not superior to SC in reducing the frequency of grade 3-4 AEs, but it did not compromise patient safety. Implementation of IOD-CAM may improve the QoL, anxiety, and emotional well-being of the patients by reducing the level of nausea, vomiting and diarrhea.. Finally, IOD-CAM potentially improves the patients' self-care, which contributes to increased treatment adherence and improved survival.

The findings of this study will be further investigated in a phase III randomized trial, including collection of qualitative data on IOD-CAM experience to demonstrate how it affects the patients and which elements of IOD-CAM are important and helpful to the patients.

\section{Declarations}

\section{Funding}

Support was received from The Idella Foundation. The study sponsor had no role in the study design; collection, analysis and interpretation of data; writing the report; or the decision to submit the manuscript for publication. 


\section{Conflicts of interest}

The authors have no conflicts of interest relevant to this article to disclose.

\section{Availability of data and material}

N/A

\section{Code availability}

$\mathrm{N} / \mathrm{A}$

\section{Author contributions}

All authors contributed to the study conception and design. Material preparation, data collection and analysis were performed by Mette Stie and Lars Henrik Jensen. The first draft of the manuscript was written by Mette Stie and all authors commented on previous versions of the manuscript. All authors read and approved the final manuscript.

\section{Ethics approval}

This study was approved by the Danish Data Protection Agency through the Region of Southern Denmark (19-4309). According to the Committee on Health Research Ethics, their approval of the study was not required (15/42744). The procedures used in this study adhere to the tenets of the Declaration of Helsinki.

\section{Consent to participate}

Informed consent was obtained from all individual participants in the study.

\section{Consent for publication}

N/A

\section{References}

1. Horneber $\mathrm{M}$ et al (2012) How many cancer patients use complementary and alternative medicine: a systematic review and metaanalysis. Integr Cancer Ther. 11(3):187-203. DOI: 
10.1177/1534735411423920.

2. Kust D et al (2016) Use of Alternative and Complementary Medicine in Patients with Malignant Diseases in High-Volume Cancer Center and Future Aspects. Acta Clinica Croatica. 55(4):585-592. DOI: https://dx.doi.org/10.20471/acc.2016.55.04.08.

3. Fremd C et al (2017) Use of complementary and integrative medicine among German breast cancer patients: predictors and implications for patient care within the PRAEGNANT study network. Archives of Gynecology \& Obstetrics. 295(5):1239-1245. DOI: 10.1007/s00404-017-4348-2.

4. Wanchai A, Armer JM, Stewart BR (2010) Complementary and alternative medicine use among women with breast cancer: a systematic review. Clinical Journal of Oncology Nursing. 14(4): E45-55. DOI: https://dx.doi.org/10.1188/10.CJON.E45-E55.

5. Nissen $\mathrm{N}$, et al (2014) The use of complementary and alternative medicine after the completion of hospital treatment for colorectal cancer: findings from a questionnaire study in Denmark. BMC Complementary \& Alternative Medicine. 14:388. DOI: https://dx.doi.org/10.1186/1472-6882-14-388.

6. Bahall M (2017) Prevalence, patterns, and perceived value of complementary and alternative medicine among cancer patients: a cross-sectional, descriptive study. BMC Complement Altern Med. 17(1):345. DOI: 10.1186/s12906-017-1853-6.

7. Towler P, Molassiotis A, Brearley SG (2013) What is the evidence for the use of acupuncture as an intervention for symptom management in cancer supportive and palliative care: an integrative overview of reviews. Support Care Cancer. 21(10):2913-23. DOI: 10.1007/s00520-013-1882-8.

8. Keyhanmehr AS et al (2018) Aromatherapy for the management of cancer complications: A narrative review. Complement Ther Clin Pract. 31:175-180. DOI: 10.1016/j.ctcp.2018.02.009.

9. Cramer $\mathrm{H}$ et al (2017) Yoga for improving health-related quality of life, mental health and cancerrelated symptoms in women diagnosed with breast cancer. Cochrane Database of Systematic Reviews. 1:CD010802. DOI: https://dx.doi.org/10.1002/14651858.CD010802.pub2.

10. Bar-Sela G et al. (2015) The effect of complementary and alternative medicine on quality of life, depression, anxiety, and fatigue levels among cancer patients during active oncology treatment: phase II study. Support Care Cancer. 23(7):1979-85. DOI: 10.1007/s00520-014-2560-1.

11. Baarts C, Pedersen IK (2009) Derivative benefits: exploring the body through complementary and alternative medicine. Sociol Health IIIn. 31(5):719-33. DOI: 10.1111/j.1467-9566.2009.01163.x.

12. Ebel MD et al (2015) Perception of cancer patients of their disease, self-efficacy and locus of control and usage of complementary and alternative medicine. Journal of Cancer Research \& Clinical Oncology. 141(8):1449-55. DOI: https://dx.doi.org/10.1007/s00432-015-1940-3.

13. Johnson SB et al (2018) Use of Alternative Medicine for Cancer and Its Impact on Survival. J Natl Cancer Inst. 110(1). DOI: 10.1093/jnci/djx145.

14. Greenlee H et al (2016) Association Between Complementary and Alternative Medicine Use and Breast Cancer Chemotherapy Initiation: The Breast Cancer Quality of Care (BQUAL) Study. JAMA oncology. 2(9):1170-1176. 
15. de Jong, FA et al (2008) Lifestyle habits as a contributor to anti-cancer treatment failure. European Journal of Cancer. 44(3):374-382. DOI: 10.1016/j.ejca.2007.12.012.

16. Davis EL et al (2012) Cancer patient disclosure and patient-doctor communication of complementary and alternative medicine use: a systematic review. Oncologist. 17(11):1475-81. DOI: https://dx.doi.org/10.1634/theoncologist.2012-0223.

17. Frenkel M, Cohen L (2014) Effective communication about the use of complementary and integrative medicine in cancer care. Journal of Alternative \& Complementary Medicine. 20(1):12-8. DOI: https://dx.doi.org/10.1089/acm.2012.0533.

18. Roter DL et al (2016) Communication predictors and consequences of Complementary and Alternative Medicine (CAM) discussions in oncology visits. Patient Educ Couns. 99(9):1519-25. DOI: 10.1016/j.pec.2016.06.002.

19. Stie M et al (2020) Open dialogue about complementary and alternative medicine (CAM) integrated in conventional oncology care, characteristics and impact. A systematic review. Patient Educ Couns. 103(11):2224-2234. DOI: 10.1016/j.pec.2020.06.003.

20. Frenkel M, Ben-Arye E, Cohen L (2010) Communication in cancer care: discussing complementary and alternative medicine. Integrative Cancer Therapies. 9(2):177-85. DOI: https://dx.doi.org/10.1177/1534735410363706.

21. Juraskova I et al (2010) Discussing complementary therapy use with early-stage breast cancer patients: exploring the communication gap. Integrative Cancer Therapies. 9(2):168-76. DOI: https://dx.doi.org/10.1177/1534735410365712.

22. Frenkel M. Cohen $L$ (2008) Incorporating complementary and integrative medicine in a comprehensive cancer center. Hematology/Oncology Clinics of North America. 22(4):727-736.

23. Chong OT (2006) An integrative approach to addressing clinical issues in complementary and alternative medicine in an outpatient oncology center. Clinical Journal of Oncology Nursing. 10(1):83-8.

24. Witt CM et al (2017) A Comprehensive Definition for Integrative Oncology. Journal of the National Cancer Institute. Monographs. 52:01. DOI: https://dx.doi.org/10.1093/jncimonographs/lgx012.

25. Weil AT (2014) Integrative Oncology. 2 ed. Oxford University Press.

26. Schofield P et al (2010) Effectively discussing complementary and alternative medicine in a conventional oncology setting: communication recommendations for clinicians. Patient Education \& Counseling. 79(2):143-51. DOI: https://dx.doi.org/10.1016/j.pec.2009.07.038.

27. Moher D et al (2010) CONSORT 2010 explanation and elaboration: updated guidelines for reporting parallel group randomised trials. BMJ. 340:c869. DOI: 10.1136/bmj.c869.

28. A.W.C.f.I.(2020) What is Integrative Medicine?

https://integrativemedicine.arizona.edu/about/definition.html. Accessed 18 January 2021

29. REDCap (2020) https://projectredcap.org/ Accessed January 2021;. 
30. Basch E et al (2014) Development of the National Cancer Institute's patient-reported outcomes version of the common terminology criteria for adverse events (PRO-CTCAE). J Natl Cancer Inst. 106(9). DOI: 10.1093/jnci/dju244.

31. The European Organisation for Research and Treatment of Cancer (EORTC) (2001) EORTC QLQ-C30 Scoring Manual https://www.eortc.org/app/uploads/sites/2/2018/02/SCmanual.pdf Acessed Juni 2020

32. Arraras $\mathrm{Jl}$ et al (2010) An international validation study of the EORTC QLQ-INFO25 questionnaire: an instrument to assess the information given to cancer patients. Eur J Cancer. 46(15):2726-38. DOI: 10.1016/j.ejca.2010.06.118.

33. Zigmond AS, Snaith RP (1983) The hospital anxiety and depression scale. Acta Psychiatr Scand. 67(6):361-70. DOI: 10.1111/j.1600-0447.1983.tb09716.x.

34. Rubinstein LV et al (2005) Design Issues of Randomized Phase II Trials and a Proposal for Phase II Screening Trials. Journal of Clinical Oncology. 23(28):7199-7206. DOI: 10.1200/jco.2005.01.149.

35. Horneber $\mathrm{M}$ et al (2018) Addressing Unmet Information Needs: Results of a Clinician-Led Consultation Service About Complementary and Alternative Medicine for Cancer Patients and Their Relatives. Integrative Cancer Therapies. 17(4):1172-1182. DOI: 10.1177/1534735418808597.

36. Frenkel $\mathrm{M}$ et al (2010) Integrative medicine consultation service in a comprehensive cancer center: findings and outcomes. Integrative Cancer Therapies. 9(3):276-83. DOI: https://dx.doi.org/10.1177/1534735410378663.

37. Münstedt K et al (2014) Wishes and Beliefs of Cancer Patients Regarding Counseling on Integrative Medicine. Breast Care. 9(6):416-420. DOI: 10.1159/000368428.

38. Molassiotis A et al (2017) MASCC/ESMO Antiemetic Guidelines: Introduction to the 2016 guideline update. Support Care Cancer. 25(1):267-269. DOI: 10.1007/s00520-016-3324-x.

39. Bossi P et al (2018) Diarrhoea in adult cancer patients: ESMO Clinical Practice Guidelines. Ann Oncol. 29(Suppl 4):iv126-iv142. DOI: 10.1093/annonc/mdy145.

40. Broom A, Adams J (2009) Oncology clinicians' accounts of discussing complementary and alternative medicine with their patients. Health: an Interdisciplinary Journal for the Social Study of Health, Illness \& Medicine. 13(3):317-36. DOI: https://dx.doi.org/10.1177/1363459308101806.

41. Klafke $\mathrm{N}$ et al (2019) The effects of an integrated supportive care intervention on quality of life outcomes in outpatients with breast and gynecologic cancer undergoing chemotherapy: Results from a randomized controlled trial. Cancer Med. 8(8):3666-3676. DOI: 10.1002/cam4.2196.

42. Shalom-Sharabi I et al (2017) Effect of a 12-week integrative oncology intervention on gastrointestinal concerns in patients with gynecological and breast cancer undergoing chemotherapy. Medical Oncology. 34(9):155. DOI: https://dx.doi.org/10.1007/s12032-017-1016-0.

43. Zwingmann $\mathrm{J}$ et al (2017) Effects of patient-centered communication on anxiety, negative affect, and trust in the physician in delivering a cancer diagnosis: A randomized, experimental study. Cancer. 123(16):3167-3175. DOI: 10.1002/cncr.30694. 
44. Radwin L (2000) Oncology patients' perceptions of quality nursing care. Res Nurs Health. 23(3):17990. DOI: 10.1002/1098-240x(200006)23:3<179::aid-nur2>3.0.co;2-t.

45. Keshet $Y$ et al (2015) Giving voice to cancer patients: assessing non-specific effects of an integrative oncology therapeutic program via short patient narratives. Psycho-Oncology. 24(2):169-74. DOI: https://dx.doi.org/10.1002/pon.3621.

\section{Tables}

Tables 1-2 are available in the Supplementary Files

\section{Figures}

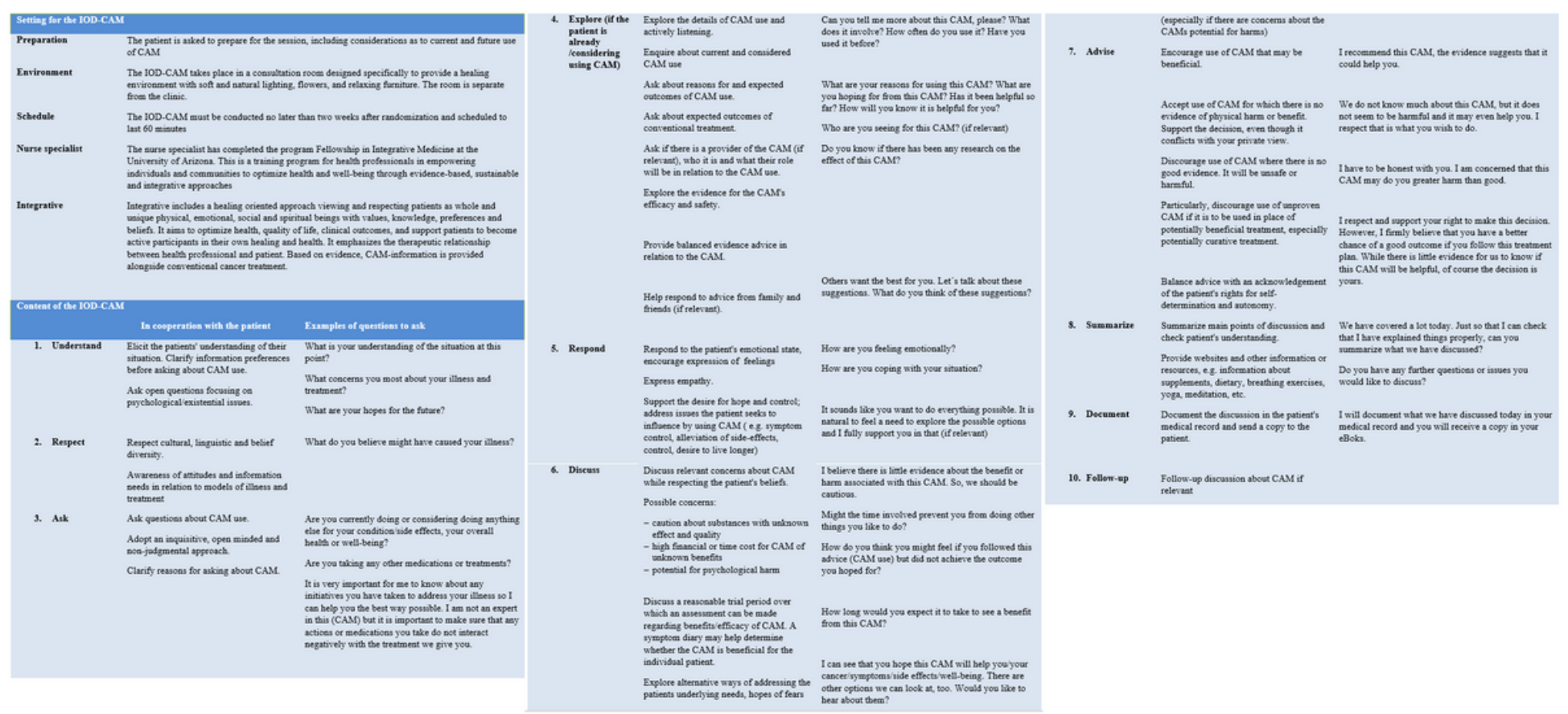

Figure 1

Guideline for Integrative open dialogue about complementary alternative medicine: IOD-CAM 


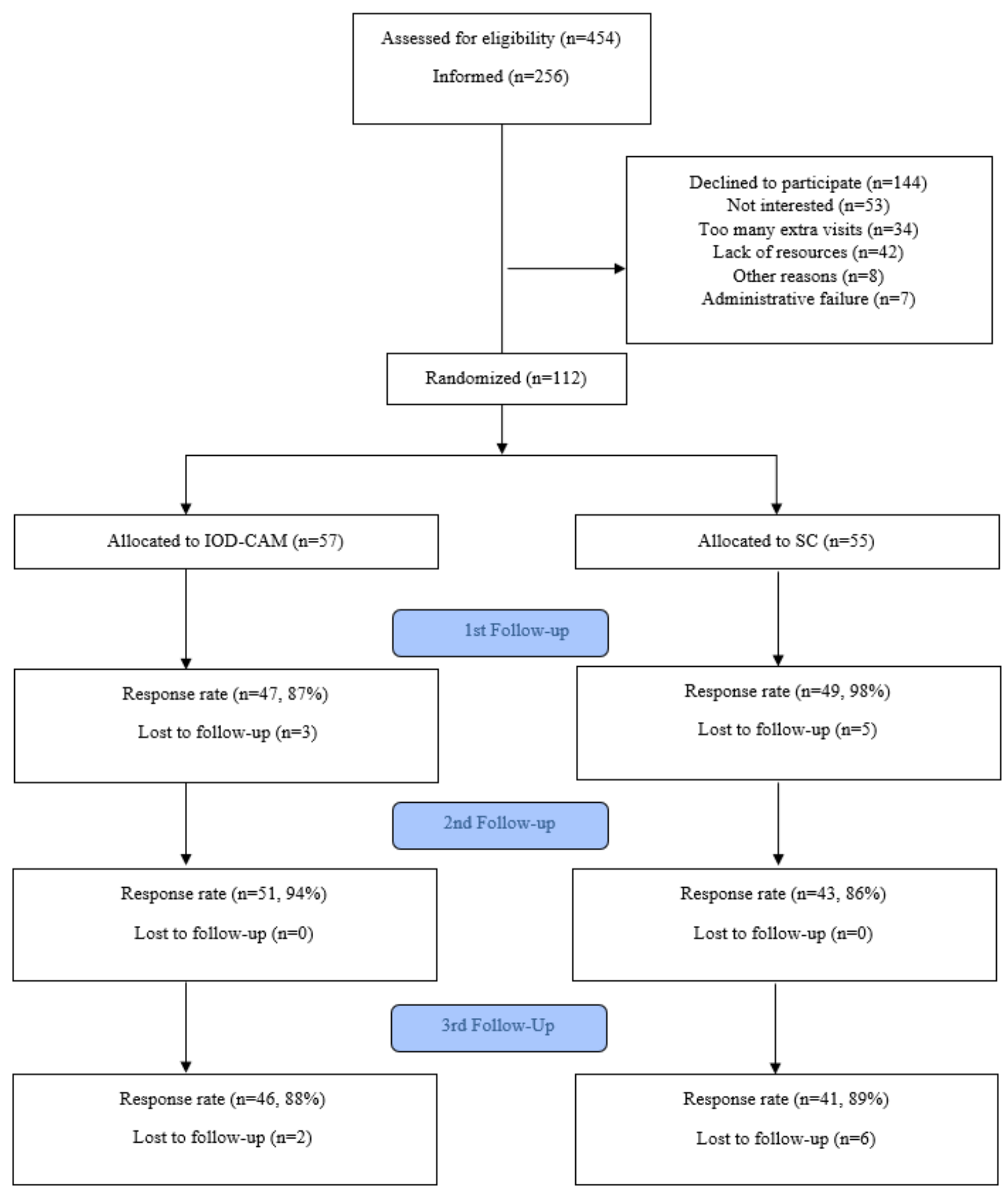

Figure 2

Flow Diagram 
Change from baseline to second follow-up (12 weeks)

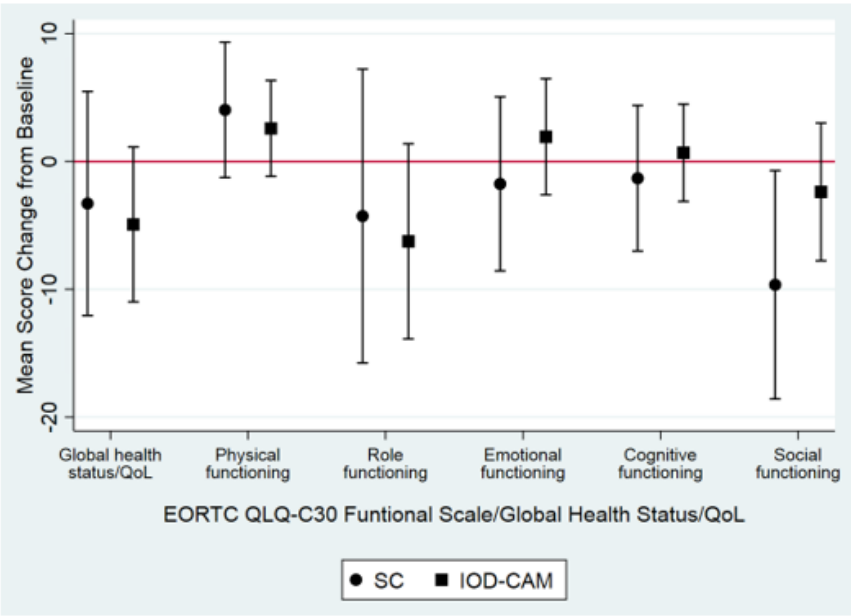

Change from baseline to third follow-up (24 weeks)

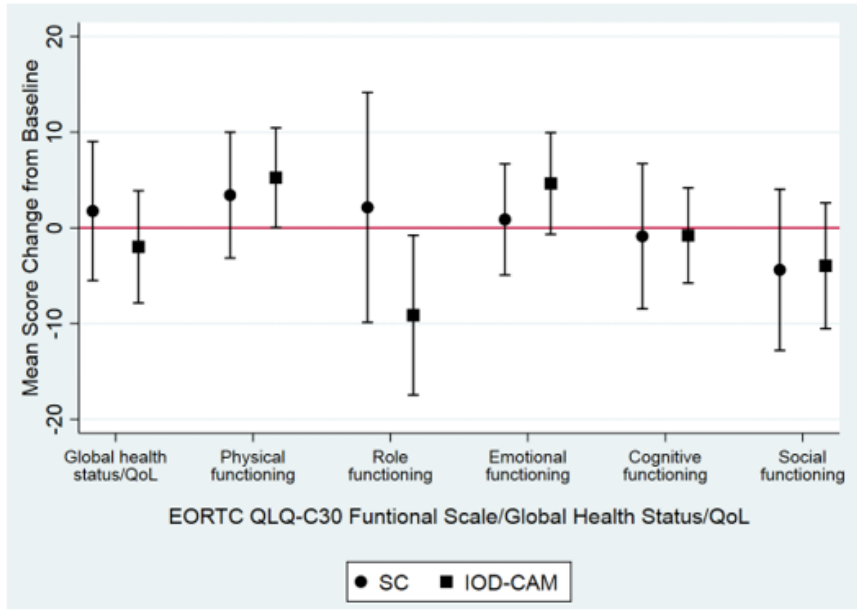

$\mathrm{SC}=$ Standard care

IOD-CAM= Integrative open dialogue about complementary alternative medicine
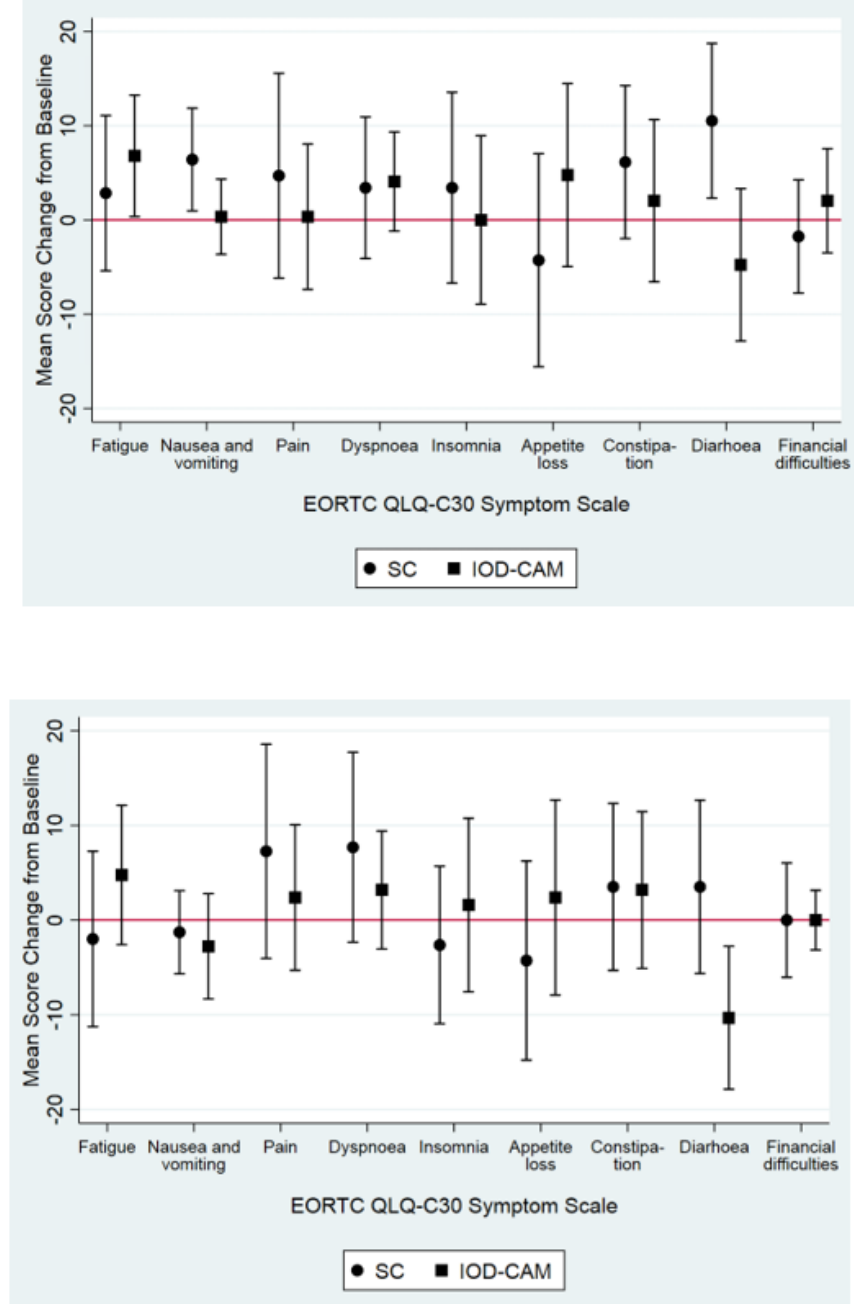

Figure 3

Quality of life 

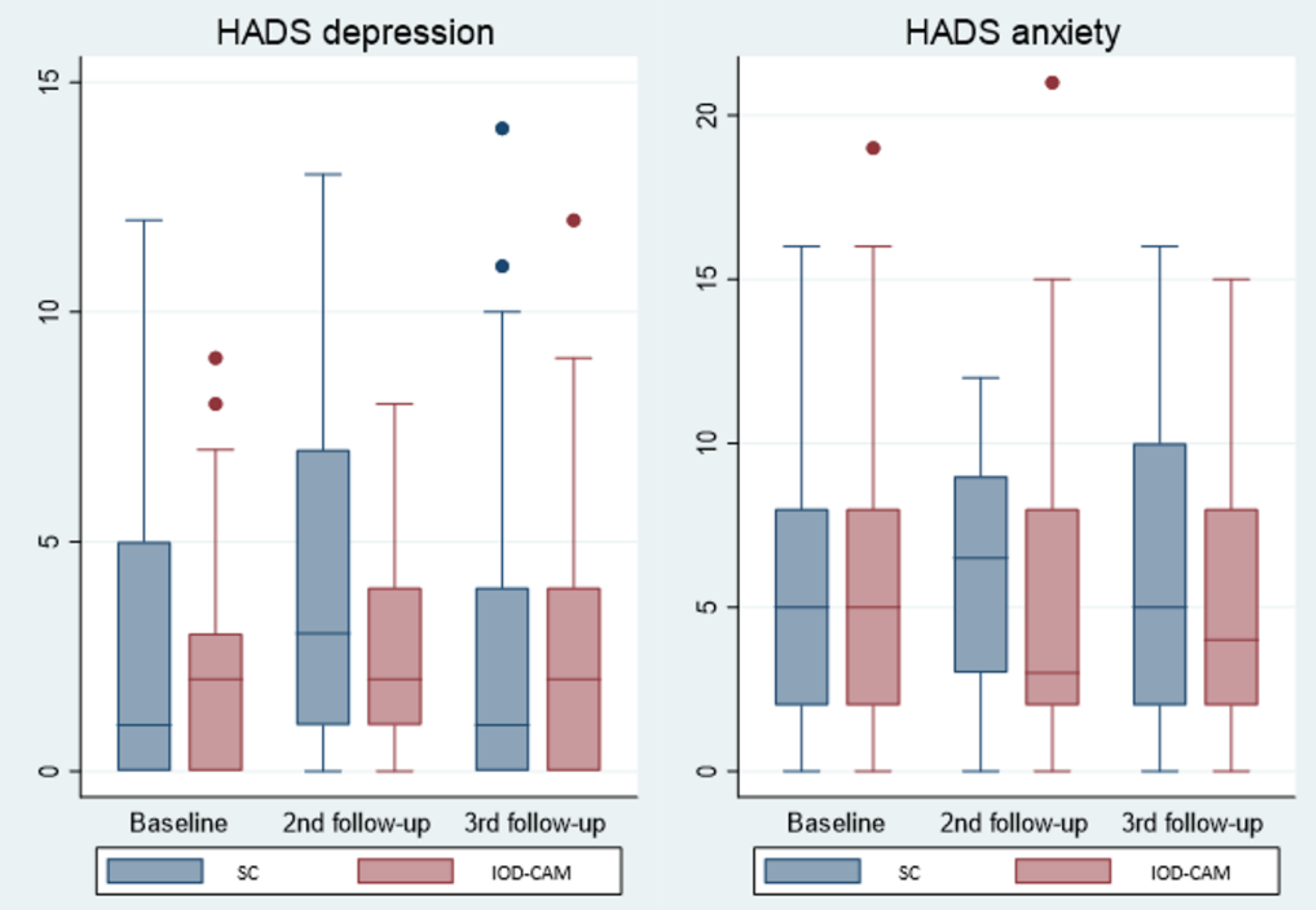

$\mathrm{SC}=$ Standard care, IOD-CAM= Integrative open dialogue about complementary alternative medicine

Figure 4

Depression and Anxiety 


\section{Global score - info25}

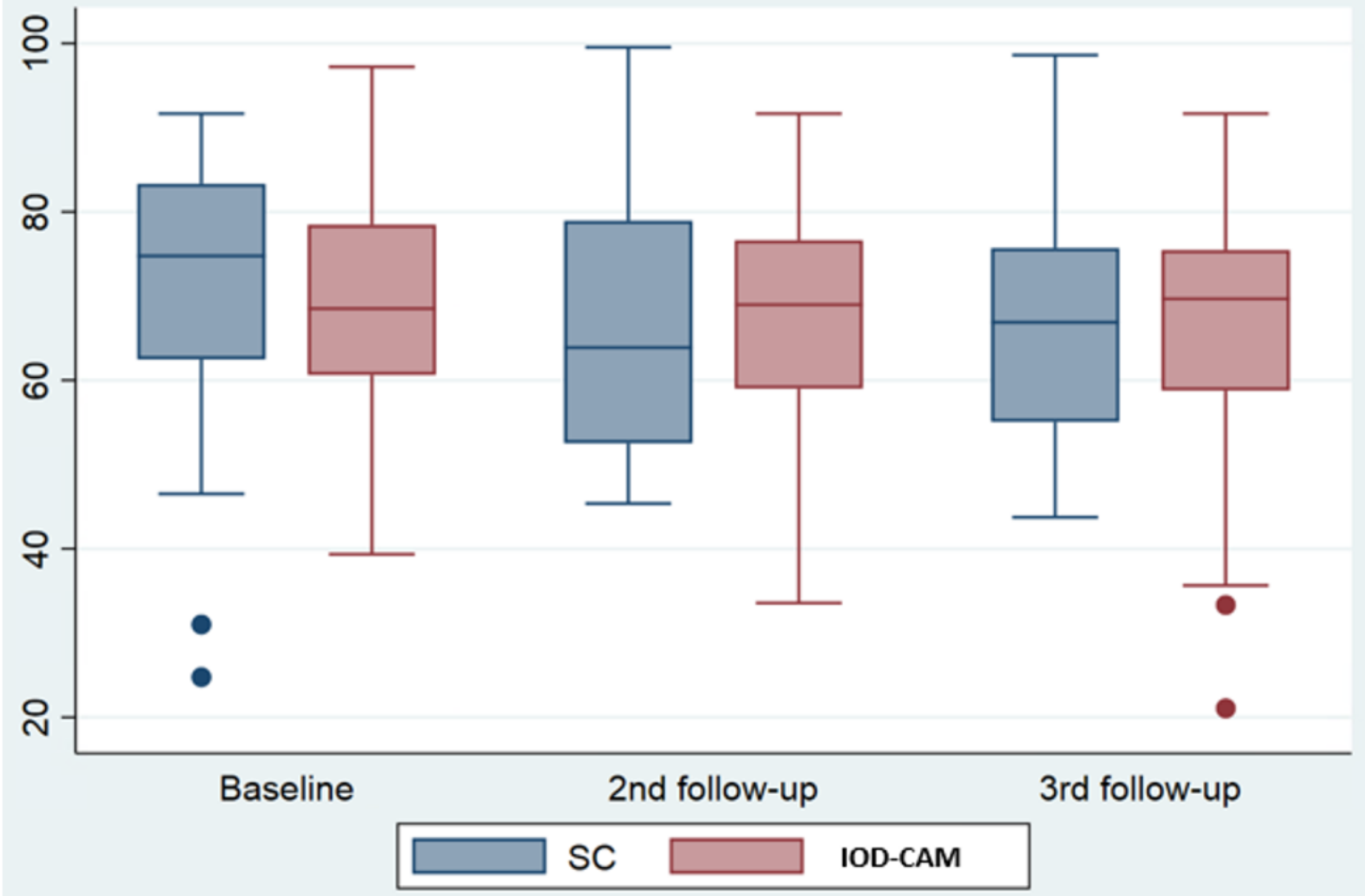

$\mathrm{SC}=\mathrm{Standard}$ care, $\mathrm{IOD}-\mathrm{CAM}=$ Integrative open dialogue about complementary alternative medicine

Figure 5

Perceived information 


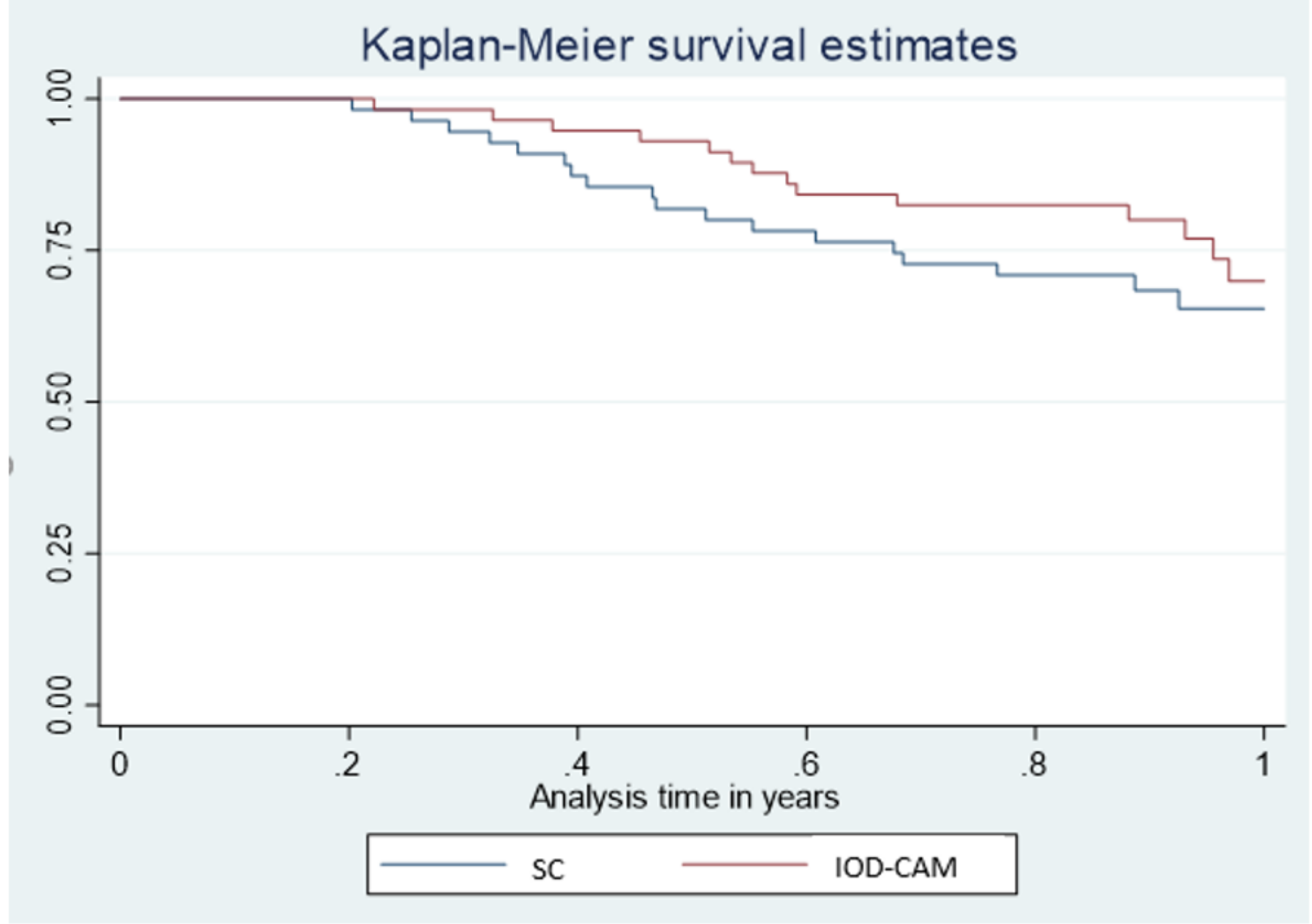

$\mathrm{SC}=$ Standard care, IOD-CAM= Integrative open dialogue about complementary alternative medicine

Figure 6

Restricted mean survival

\section{Supplementary Files}

This is a list of supplementary files associated with this preprint. Click to download.

- Table1baselinecharacteristics.docx

- Table2AdverseEventsFrequency.docx 